\title{
Resistotyping of Proteus mirabilis and a comparison with other methods of typing
}

\author{
I. M. KASHBUR ${ }^{1}$, R. H. GEORGE, AND G. A. J. AYLIFFE \\ From the Department of Virology, University of Birmingham Medical School
}

SYNOPSIS Resistotyping of $P$. mirabilis using 10 compounds is reported. The method was tested for reproducibility and specificity and results were compared with those obtained by serological, bacteriophage, and proticine typing methods and the Dienes test. The possible relationship between resistance to the chemicals used in the test and antibiotics was also studied.

The method was found to be simple, reproducible, show good specificity, and compare favourably with other typing methods. No linkage between resistance to the chemicals and antibiotic resistance was detected.

Proteus mirabilis is a normal commensal of the intestinal tract and can cause bacteraemia and infection of the urinary tract, of wounds, of burns, and of the meninges. The incidence of severe infections due to this organism appears to be increasing (Finland, 1960; Lewis and Feckety, 1969) and outbreaks of infection are occasionally reported (Adler, Burke, Martin, and Finland, 1971). Although the Dienes test is a useful method for differentiating strains in a routine laboratory, other methods are sometimes required to recognize cross-infection or for routine typing of strains. The methods available, namely, serological, phage, and proticine, are either not sufficiently discriminating or are not readily available. Another method, resistotyping, depending on the selective toxicity of antibacterial compounds other than antibiotics, has been described for typing of E. coli and Sh. Sonnei (Elek and Higney, 1970; Elek, Davies, and Miles, 1973). In the present study, a set of 10 chemicals was chosen with varying inhibitory activity against strains of Proteus mirabilis, and a suitable medium was developed for reading the results. The method was compared with other typing methods.

\section{Materials and Methods}

STRAINS

Twenty strains of $P$. mirabilis shown to be different

${ }^{1}$ Present address: Department of Microbiology, School of Medicine, University of Benghazi, Libya

${ }^{2}$ Hospital Infection Research Laboratory, Summerfield Hospital, Birmingham 18

Received for publication 20 March 1974. by the Dienes test (Skirrow, 1969) were selected initially as control strains from a number of routine laboratory isolates. These strains proved to be satisfactory and were used as controls in all the tests. Eighty-nine strains of unknown type isolated from a routine laboratory and $\mathbf{1 1 5}$ other strains of known phage, serological, and proticine type were resistotyped. Strains isolated from the faeces and urine of 18 patients and the umbilicus and faeces of two babies were also typed.

\section{MEDIA}

Tryptose (Difco) agar with $0.5 \%$ sodium taurocholate was made up in Tris buffer (BDH) at $\mathrm{pH} 7.6$ and used in all tests. Tryptose agar was used at a concentration $10 \%$ higher than recommended by the manufacturer. Triphenyl tetrazolium chloride (TTC) $0.2 \%$ was used as an indicator and was added to the medium with the required chemical. Peptone water (Oxoid) was used for growing strains before inoculation of plates.

SELECTION OF ANTIBACTERIAL COMPOUNDS One hundred and seven chemical compounds, both inorganic and organic, including some dyes and disinfectants, were tested for activity against the control strains. Antibiotics, phenolic disinfectants, and highly volatile compounds were excluded. Screening by the gradient plate method of Szybalski (1952) was initially used but was found to be unnecessarily time consuming and was replaced by a plate dilution method. Dilutions ranging from $10^{-1}$ to $10^{-6}$ were prepared from $10 \% \mathrm{w} / \mathrm{v}$ stock solutions. One $\mathrm{ml}$ of 
each dilution and $1 \mathrm{ml}$ of $0.2 \%$ TTC solution were added to $18 \mathrm{ml}$ of melted agar, mixed, and plates were poured. After drying for 20 minutes at $45^{\circ} \mathrm{C}$ plates were inoculated using a phage-typing multiinoculator fitted with either 25 loops or 25 straight wires for stab cultures. Cultures of the unknown strains and controls grown in peptone water for 18 hours were diluted $10^{-3}$ for the loop or $10^{-2}$ for the stab technique. After inoculation plates were incubated at $37^{\circ} \mathrm{C}$ for 18 hours and the patterns of inhibition of the control strains for each chemical determined.

Fifty of the compounds tested showed no antibacterial activity and the remaining 57 were titrated in smaller steps around the concentration indicated by initial screening. Ten compounds (table I) showed satisfactory discrimination, easy interpretation, and good reproducibility of patterns of inhibition of control strains and were chosen as the basis of the typing methods. These compounds were finally prepared at concentrations, which, when added to $19 \mathrm{ml}$ of agar, produced optimal differential activity on the control strains.

\section{TECHNIQUE OF RESISTOTYPING}

The 10 standard chemicals were dissolved in sterile distilled water to give the concentrations shown in table I. Triphenyl tetrazolium chloride was added to these solutions to give a concentration of $0.2 \%$. An exception was the periodate solution since precipitation occurred on addition of TTC, which was added separately to the medium. Sterilization of the chemicals by heat tended to alter their properties and since no contamination of plates was noted with unsterilized solutions the stock solutions were not sterilized.

The medium was dispensed in 19-ml volumes in tightly sealed McCartney bottles and autoclaved. Five different concentrations of each of the stock chemical solutions were used in the test : $0 \cdot 8,0.9,1 \cdot 0$, $1 \cdot 1$, and $1.2 \mathrm{ml}$ volumes were added to the melted agar medium, mixed thoroughly, and plates were poured. Plates were dried and inoculated with over- night broth cultures using either the loop or the stab method. Control plates without chemicals were also included. In the stab method, 25 strains were inoculated into the agar, the plate was offset slightly and a further 25 strains were inoculated into the agar. Plates were dried for 15 minutes and then incubated for 18 hours.

\section{READING THE TEST}

The plates containing the concentration of the chemical showing the typical pattern of inhibition of the control strains were chosen for recording patterns of the unknown strains (table II). Strains showing confluent growth were regarded as resistant and assigned the appropriate capital letter, eg, A, B, C, etc, and strains showing non-confluent growth but more than 15 colonies were recorded in parentheses, eg, (A). Strains showing complete inhibition or less than 15 colonies were described as sensitive for that chemical and not recorded. Tests were only accepted when confluent growth was recorded on the control plates.

\section{ANTIBIOTIC SENSITIVITY PATTERNS AND} RESISTANCE TRANSFER

Diagnostic sensitivity test agar (Oxoid) was flooded with diluted broth cultures of strains diluted to give an almost confluent growth. After drying the following discs (Mast Laboratory) were applied: ampicillin (Ap) $10 \mu \mathrm{g}$, cephaloridine (Cp) $5 \mu \mathrm{g}$, carbenicillin (Py) $100 \mu \mathrm{g}$, chloramphenicol (C) 25 $\mu \mathrm{g}$, gentamicin (Gn) $10 \mu \mathrm{g}$, kanamycin (Kn) $30 \mu \mathrm{g}$, sulphonamide (S) $200 \mu \mathrm{g}$, and tetracycline (T) $25 \mu \mathrm{g}$.

Resistance to $\mathrm{Ap}, \mathrm{Cp}, \mathrm{Py}$, and $\mathrm{Kn}$ were transferred from a non-swarming to five swarming strains of $\boldsymbol{P}$. mirabilis sensitive to the four antibiotics of different resistotype, using the technique of Datta (1968). Recipient strains were selected as swarming organisms on a plate containing kanamycin $10 \mu \mathrm{g} / \mathrm{ml}$. Strains were resistotyped before and after transfer of antibiotic resistance.

\begin{tabular}{llll}
\hline Reference Letter & Substance & Concentrations $(\% w / v)$ & $0 \cdot 2 \%$ TTC Added \\
\hline A & Sodium hydrogen selenite & 15 & + \\
B & Cetrimide & 8 & + \\
C & Malachite green & 0.065 & + \\
D & Acriflavine & 0.4 & + \\
E & Benzalkonium chloride & 5 & + \\
F & Orcinol & 5 & + \\
G & Sodium periodate & 1.5 & + \\
H & Chlorhexidine diacetate & $\mathbf{0 . 5}$ & + \\
I & Irgasan 'CH 3565' (Geigy) & $\mathbf{0 . 0 0 2}$ & + \\
K & Potassium tellurite & $\mathbf{0 . 0 3}$ & + \\
\hline
\end{tabular}

Table I Stock solutions for resistotyping

${ }^{2}+=$ TTC added to stock solution, $-=$ TTC added directly to agar 


\begin{tabular}{|c|c|c|c|c|c|c|c|c|c|c|}
\hline \multirow[t]{2}{*}{ Strain } & \multicolumn{10}{|c|}{ Resistotype (see table I) } \\
\hline & $A$ & $B$ & $C$ & $D$ & $\boldsymbol{E}$ & $F$ & $G$ & $\boldsymbol{H}$ & $I$ & $K^{1}$ \\
\hline P1 & + & + & $(-)$ & + & $(+)$ & + & + & + & - & + \\
\hline P2 & + & - & + & + & - & + & + & + & $(+)$ & $(+)$ \\
\hline P3 & + & + & + & + & + & $(-)$ & + & $(+)$ & + & + \\
\hline P4 & + & + & $(+)$ & + & + & + & + & $(+)$ & $(+)$ & $\tau$ \\
\hline P5 & - & $(+)$ & $(+)$ & $(+)$ & - & $(-)$ & $(-)$ & - & - & - \\
\hline P6 & - & + & - & - & $(+)$ & - & $(+)$ & $(+)$ & $(+)$ & + \\
\hline P7 & + & + & $(+)$ & + & + & + & + & $(-)$ & $(+)$ & - \\
\hline P8 & + & + & $(+)$ & + & + & - & + & $(-)$ & $(+)$ & $(+)$ \\
\hline P9 & + & - & + & + & - & + & + & $(+)$ & $(+)$ & + \\
\hline P10 & + & $(+)$ & + & + & - & $(+)$ & + & - & $(+)$ & + \\
\hline P11 & - & - & + & + & - & + & - & $(-)$ & + & + \\
\hline P12 & + & + & + & + & + & + & + & + & $(+)$ & $(-)$ \\
\hline P13 & + & - & + & - & - & + & + & - & $(+)$ & - \\
\hline P14 & $(+)$ & + & $(-)$ & + & + & + & + & - & + & - \\
\hline P15 & + & + & + & + & + & $(+)$ & + & + & + & + \\
\hline P16 & + & + & + & + & + & + & - & + & + & $(+)$ \\
\hline P17 & - & $(+)$ & - & - & $(-)$ & + & + & $(+)$ & $(+)$ & - \\
\hline P18 & $(-)$ & - & + & + & - & + & + & + & $(+)$ & $(-)$ \\
\hline P19 & + & - & + & + & $(+)$ & + & + & + & + & $(+)$ \\
\hline $\mathbf{P 2 0}$ & + & + & $(+)$ & + & + & + & + & + & $(+)$ & $(+)$ \\
\hline
\end{tabular}

Table II Growth pattern of P. mirabilis control strains determining the concentration for recording the resistotype of unknown strains

$+=$ Confluent growth, $(+)=$ non-confluent growth over 15 colonies (or fine confluent growth), $(-)=$ less than 15 colonies,

- = no growth.

\section{Results}

REPRODUCIBILITY AND INTERPRETATION

Thirty-three strains were tested on four occasions. Labelling of the strains was varied for each test, so that the operator was unaware of the identity of the strains. Seventeen strains showed complete agreement on all occasions and 15 varied by only one degree of resistance, ie, between complete and partial resistance. One strain varied by one major reaction. Strains assigned the same letters irrespective of parentheses were considered to be similar, eg, A C D F G H and A (C) D (F) G H, and also strains differing by a reaction of moderate resistance to one chemical, eg, A B C D and A B C D (E). Strains that differ by one major reaction or more, eg, A B C D and $A$ B C, were regarded as different, as were strains differing in moderate resistance to two or more chemicals, eg, A B C D and A B C D (E) (F). This interpretation was confirmed in further tests on epidemiologically related strains. Continuous testing of controls also confirmed this degree of reproducibility.

TESTS ON CONSECUTIVE ISOLATES

Fifty-six types were differentiated in the 86 routine isolates examined. Ten $(11.2 \%)$ of the strains were of one type (A B C D E F G H), five $(5.6 \%)$ were of another type (A B D E F G H), and on 11 occasions two strains of a similar type were identified; 39 isolates were of single types.

COMPARISON WITH OTHER TYPING METHODS

Fifty-six strains previously typed by other methods

\begin{tabular}{|c|c|c|c|c|c|c|c|c|c|c|c|c|c|c|c|}
\hline \multirow[t]{2}{*}{ Patient } & \multirow{2}{*}{$\begin{array}{l}\text { Dienes } \\
\text { Type }\end{array}$} & \multicolumn{10}{|c|}{ Resistotype } & \multicolumn{2}{|c|}{ Serotype } & \multirow[t]{2}{*}{ Phage Type } & \multirow{2}{*}{$\begin{array}{l}\text { Proticine } \\
\text { Type }\end{array}$} \\
\hline & & $A$ & $B$ & $C$ & $D$ & $E$ & $F$ & $G$ & $H$ & $I$ & $\boldsymbol{K}$ & $O$ & $\boldsymbol{H}$ & & \\
\hline $\begin{array}{l}1 \\
2 \\
3\end{array}$ & $\begin{array}{l}\text { T2 } \\
\text { T2 } \\
\text { T2 }\end{array}$ & - & $\begin{array}{l}\mathbf{B} \\
\mathbf{B} \\
\mathbf{B}\end{array}$ & $\begin{array}{c}\mathrm{C} \\
\mathrm{C} \\
\text { (C) }\end{array}$ & $\begin{array}{c}\mathrm{D} \\
\text { (D) } \\
\mathrm{D}\end{array}$ & $\begin{array}{c}\mathbf{E} \\
(\mathbf{E}) \\
\mathbf{E}\end{array}$ & $\begin{array}{c}\text { (F) } \\
\text { (F) } \\
F\end{array}$ & $\begin{array}{l}\mathbf{G} \\
\mathbf{G} \\
\mathbf{G}\end{array}$ & $\overline{-}$ & (I) & $\begin{array}{l}\mathbf{K} \\
\mathbf{K} \\
\mathbf{K}\end{array}$ & $\begin{array}{r}3 \\
-\end{array}$ & $\frac{1}{-}$ & $\begin{array}{l}2 / 8 \\
-\end{array}$ & $\begin{array}{l}5 \\
5 \\
5\end{array}$ \\
\hline $\begin{array}{l}4 \\
4\end{array}$ & $\begin{array}{l}\text { T9 } \\
\text { T9 }\end{array}$ & $\begin{array}{l}\mathbf{A} \\
\mathbf{A}\end{array}$ & $\begin{array}{l}\mathbf{B} \\
\mathbf{B}\end{array}$ & $\underline{\mathrm{C}}$ & $\begin{array}{l}\mathbf{D} \\
\mathbf{D}\end{array}$ & $\begin{array}{c}E \\
(E)\end{array}$ & $\begin{array}{l}\mathbf{F} \\
\mathbf{F}\end{array}$ & $\begin{array}{l}\mathbf{G} \\
\mathbf{G}\end{array}$ & $\underline{\mathbf{H}}$ & $\begin{array}{l}\text { (I) } \\
\text { (I) }\end{array}$ & $\overline{\mathbf{K}}$ & $\begin{array}{l}13 \\
30\end{array}$ & $\begin{array}{l}2 \\
2\end{array}$ & $\begin{array}{l}26 w^{2} \\
26 w\end{array}$ & $\begin{array}{l}1 \\
1\end{array}$ \\
\hline $\begin{array}{l}5 \\
5 \\
5\end{array}$ & $\begin{array}{l}\text { T10 } \\
\text { T10 } \\
\text { T10 }\end{array}$ & - & $\frac{(B)}{(B)}$ & $\frac{\mathrm{C}}{(\mathrm{C})}$ & $\begin{array}{l}\mathbf{D} \\
\mathbf{D} \\
\mathbf{D}\end{array}$ & - & $\begin{array}{l}\mathbf{F} \\
\mathbf{F} \\
\mathbf{F}\end{array}$ & $\begin{array}{c}\text { (G) } \\
- \\
-\end{array}$ & $\begin{array}{l}\mathbf{H} \\
\mathbf{H} \\
\mathbf{H}\end{array}$ & $\begin{array}{l}\text { (I) } \\
\text { I }\end{array}$ & $\begin{array}{l}- \\
(\mathrm{K}) \\
-\end{array}$ & $\begin{array}{l}13 \\
13 \\
30\end{array}$ & $\frac{-}{1}$ & $\begin{array}{l}26 \\
\mathrm{NT}^{2} \\
26\end{array}$ & $\begin{array}{l}\text { NT } \\
\text { NT } \\
\text { NT }\end{array}$ \\
\hline $\begin{array}{l}6 \\
6\end{array}$ & $\begin{array}{l}\text { T12 } \\
\text { T12 }\end{array}$ & $\begin{array}{l}\mathbf{A} \\
\mathbf{A}\end{array}$ & $\begin{array}{c}\text { B } \\
\text { (B) }\end{array}$ & $\underline{\mathrm{C}}$ & $\bar{D}$ & (E) & $\begin{array}{l}\mathbf{F} \\
\mathbf{F}\end{array}$ & $\begin{array}{l}\mathbf{G} \\
\mathbf{G}\end{array}$ & - & $\underline{\text { (I) }}$ & - & $\begin{array}{l}\text { NT } \\
\text { NT }\end{array}$ & $\begin{array}{l}1 \\
1\end{array}$ & $\begin{array}{l}19 / 28 \\
8 / 19 / 28 / 29 / 31\end{array}$ & $\begin{array}{l}\text { NT } \\
\text { NT }\end{array}$ \\
\hline $\begin{array}{l}7 \\
7\end{array}$ & $\begin{array}{l}\text { T20 } \\
\text { T20 }\end{array}$ & $\begin{array}{l}\mathbf{A} \\
\mathbf{A}\end{array}$ & $\begin{array}{l}\mathbf{B} \\
\mathbf{B}\end{array}$ & - & $\underline{\mathbf{D}}$ & $\begin{array}{l}\mathbf{E} \\
\mathbf{E}\end{array}$ & $\begin{array}{l}\mathbf{F} \\
\mathbf{F}\end{array}$ & $\begin{array}{l}\mathbf{G} \\
\mathbf{G}\end{array}$ & - & $\mathbf{I}$ & $\begin{array}{l}(\mathbf{K}) \\
(\mathbf{K})\end{array}$ & $\begin{array}{l}\text { NT } \\
\text { NT }\end{array}$ & $\begin{array}{l}4 \\
4\end{array}$ & $\begin{array}{l}8 / 19 / 20 / 28 / 31 \\
8 / 19 / 20 / 27 / 28 / 29 / 31\end{array}$ & $\begin{array}{l}\text { NT } \\
2\end{array}$ \\
\hline
\end{tabular}

Table III Groups of P. mirabilis of the same Dienes type but different by resistotyping and other typing methods ${ }^{1} \mathrm{w}=$ weak inhibition by phage; ${ }^{2} \mathrm{NT}=$ not typable. 
were typed by the loop inoculation method. Fifteen types could be differentiated serologically; three strains were not typable. Bacteriophage typing differentiated 17 types; 15 strains were not typable, and bacteriocine typing differentiated seven types with 11 non-typable strains. The Dienes test showed that these organisms belonged to 32 different types, whereas the resistotype method differentiated 36 types. Some types shown to be different by the resistotyping method but identical by the Dienes test were also shown to be different by one or more of the other methods (table III). Similarly, a few types not differing by total resistance to one chemical or moderate resistance to two chemicals could be shown to be different using other methods (table IV).

A further 33 strains previously typed by other methods were typed by the stab inoculation method. Twenty-one types could be differentiated serologically. Bacteriophage typing differentiated 10 types; three were non-typable strains and bacteriocine typing differentiated nine types; two were nontypable strains. The Dienes test showed that these organisms belonged to 27 different types, whereas resistotyping differentiated 28 types.

A comparison of typing methods on organisms from different sites on the same patient or taken at different times showed similar patterns. Strains from different sites on three patients are shown in table V.

\section{TESTS ON PAIRED ORGANISMS}

Sixteen faeces and urine strains were shown to be identical and two were different both by the Dienes test and by resistotyping. Two pairs of umbilical and faecal strains were shown to be identical by both methods.

\section{RELATIONSHIP BETWEEN ANTIBIOTIC}

RESISTANCE AND THE RESISTOTYPE

The acquisition of the $R$ factor determining resistance to Ap, $\mathrm{Cp}, \mathrm{Py}$, and $\mathrm{Kn}$ was not associated with any change in resistotype. There was also no correlation between resistance to ampicillin or other antibiotics and the resistotype.

\begin{tabular}{|c|c|c|c|c|c|c|c|c|c|c|c|c|c|c|c|}
\hline \multirow[t]{2}{*}{ Patient } & \multicolumn{10}{|c|}{ Resistotype } & \multirow{2}{*}{$\begin{array}{l}\text { Dienes } \\
\text { Type }\end{array}$} & \multicolumn{2}{|c|}{ Serology } & \multirow[t]{2}{*}{ Phage Type } & \multirow{2}{*}{$\begin{array}{l}\text { Proticine } \\
\text { Type }\end{array}$} \\
\hline & $A$ & $B$ & $C$ & $D$ & $E$ & $\boldsymbol{F}$ & $G$ & $H$ & $I$ & $\boldsymbol{K}$ & & 0 & $\boldsymbol{H}$ & & \\
\hline $\begin{array}{l}8 \\
4\end{array}$ & $\begin{array}{l}\mathbf{A} \\
\mathbf{A}\end{array}$ & $\begin{array}{l}\mathbf{B} \\
\mathbf{B}\end{array}$ & $\begin{array}{l}\text { C } \\
\text { C }\end{array}$ & $\begin{array}{l}\mathbf{D} \\
\mathbf{D}\end{array}$ & $\begin{array}{l}\mathbf{E} \\
\mathbf{E}\end{array}$ & $\begin{array}{l}\mathbf{F} \\
\mathbf{F}\end{array}$ & $\begin{array}{l}\mathbf{G} \\
\mathbf{G}\end{array}$ & $\stackrel{(\mathbf{H})}{\mathbf{H}}$ & $\begin{array}{c}\text { I } \\
\text { (I) }\end{array}$ & - & $\begin{array}{l}\text { T4 } \\
\text { T9 }\end{array}$ & $\begin{array}{r}3 \\
13\end{array}$ & $\begin{array}{l}2 \\
2\end{array}$ & $\begin{array}{l}8 / 9 / 19 / 20 / 27 / 28 / 29 / 31 \\
26 \mathrm{w}\end{array}$ & $\begin{array}{l}4 \\
1\end{array}$ \\
\hline $\begin{array}{l}9 \\
4 \\
5\end{array}$ & $\begin{array}{l}\text { A } \\
\text { A } \\
\text { A) }\end{array}$ & $\begin{array}{l}\text { B } \\
\mathbf{B} \\
\mathbf{B}\end{array}$ & $\begin{array}{l}\text { C } \\
\mathbf{C} \\
\mathbf{C}\end{array}$ & $\begin{array}{l}\text { (D) } \\
\text { D } \\
\text { D }\end{array}$ & $\begin{array}{c}E \\
(E) \\
E\end{array}$ & $\begin{array}{l}\mathbf{F} \\
\mathbf{F} \\
\mathbf{F}\end{array}$ & $\begin{array}{l}\mathbf{G} \\
\mathbf{G} \\
\mathbf{G}\end{array}$ & E & $\begin{array}{l}\mathbf{I} \\
\mathbf{I} \\
\mathbf{I}\end{array}$ & $\begin{array}{l}\overline{(\bar{K})} \\
(\mathbf{K})\end{array}$ & $\begin{array}{l}\text { T11 } \\
\text { T9 } \\
\text { T21 }\end{array}$ & $\begin{array}{l}23 \\
30 \\
\text { NT }\end{array}$ & $\begin{array}{l}1 \\
2 \\
\text { NT }\end{array}$ & $\begin{array}{l}19 / 27 / 28 \\
26 w^{1} \\
N^{2}\end{array}$ & $\begin{array}{l}2 \\
1 \\
\text { NT }\end{array}$ \\
\hline $\begin{array}{l}10 \\
11 \\
12\end{array}$ & $\begin{array}{c}\mathbf{A} \\
\mathbf{A} \\
\text { (A) }\end{array}$ & $\begin{array}{l}\mathbf{B} \\
\mathbf{B} \\
\mathbf{B}\end{array}$ & $\begin{array}{l}\text { (C) } \\
\text { C } \\
\text { C }\end{array}$ & $\frac{(D)}{(D)}$ & $\begin{array}{c}E \\
(E) \\
E\end{array}$ & - & $\begin{array}{l}\mathbf{G} \\
\mathbf{G} \\
\mathbf{G}\end{array}$ & $\bar{z}$ & $\begin{array}{l}\mathbf{I} \\
\mathbf{I} \\
\mathbf{I}\end{array}$ & $\begin{array}{l}\mathbf{K} \\
\mathbf{K} \\
\mathbf{K}\end{array}$ & $\begin{array}{l}\text { T15 } \\
\text { T13 } \\
\text { T30 }\end{array}$ & $\begin{array}{l}\text { NT } \\
\text { NT } \\
-\end{array}$ & $\begin{array}{l}1 \\
1 \\
-\end{array}$ & $\begin{array}{l}19 / 27 / 28 \\
19 / 27 / 28 \\
-\end{array}$ & $\begin{array}{l}1 \\
1 \\
5\end{array}$ \\
\hline $\begin{array}{r}7 \\
43 \\
11\end{array}$ & $\begin{array}{l}\mathbf{A} \\
\mathbf{A} \\
\mathbf{A}\end{array}$ & $\begin{array}{c}\text { B } \\
\text { B } \\
\text { (B) }\end{array}$ & - & $\begin{array}{l}\text { D } \\
\text { D } \\
\text { D }\end{array}$ & $\begin{array}{l}\text { (E) } \\
\text { (E) } \\
\mathrm{E}\end{array}$ & $\begin{array}{l}F \\
F \\
F\end{array}$ & $\begin{array}{l}\mathbf{G} \\
\mathbf{G} \\
\mathbf{G}\end{array}$ & $\bar{z}$ & $\begin{array}{l}\text { I } \\
\text { (I) } \\
\text { I }\end{array}$ & $\begin{array}{c}(\mathbf{K}) \\
\mathbf{K} \\
\mathbf{K}\end{array}$ & $\begin{array}{l}\text { T20 } \\
\text { T9 } \\
\text { T25 }\end{array}$ & $\begin{array}{l}\text { NT } \\
30 \\
-\end{array}$ & $\begin{array}{l}4 \\
2 \\
-\end{array}$ & $\begin{array}{l}8 / 19 / 20 / 28 \\
26 w \\
-\end{array}$ & $\begin{array}{l}2 \\
1 \\
1\end{array}$ \\
\hline $\begin{array}{l}4 \\
3\end{array}$ & $\begin{array}{c}\mathrm{A} \\
(\mathrm{A})\end{array}$ & $\begin{array}{l}\text { B } \\
\mathbf{B}\end{array}$ & $\begin{array}{l}\text { (C) } \\
\text { (C) }\end{array}$ & $\begin{array}{l}\text { D } \\
\text { D }\end{array}$ & $\begin{array}{l}\mathrm{E} \\
\mathrm{E}\end{array}$ & $\begin{array}{l}\mathbf{F} \\
\mathbf{F}\end{array}$ & $\mathbf{G}$ & $\begin{array}{l}\mathbf{H} \\
\mathbf{H}\end{array}$ & - & $\begin{array}{l}\mathbf{K} \\
\mathbf{K}\end{array}$ & $\begin{array}{l}\text { T29 } \\
\text { T2 }\end{array}$ & - & - & - & $\begin{array}{l}4 \\
5\end{array}$ \\
\hline
\end{tabular}

Table IV Groups of $\mathrm{P}$. mirabilis of the same resistotype but different by other typing methods ${ }^{1} \mathrm{~W}=$ weak inhibition by phage; ${ }^{2} \mathrm{NT}_{*}^{*}=$ not typable.

\begin{tabular}{|c|c|c|c|c|c|c|c|c|}
\hline \multirow[t]{2}{*}{ Patient } & \multirow[t]{2}{*}{ Source } & \multicolumn{2}{|c|}{ Serology } & \multirow{2}{*}{$\begin{array}{l}\text { Proticine } \\
\text { Type }\end{array}$} & \multirow[t]{2}{*}{ Phage Type } & \multirow[t]{2}{*}{ Resistotype } & \multirow[t]{2}{*}{ Dienes Type } & \multirow{2}{*}{$\begin{array}{l}\text { Date of } \\
\text { Isolation }\end{array}$} \\
\hline & & $O$ & $\boldsymbol{H}$ & & & & & \\
\hline $\mathbf{A}$ & $\begin{array}{l}\text { Nose } \\
\text { Bed } \\
\text { Sacral sore } \\
\text { Bed }\end{array}$ & $\begin{array}{l}23 \\
23 \\
26 \\
26\end{array}$ & $\begin{array}{l}1 \\
1 \\
1 \\
1\end{array}$ & $\begin{array}{l}2 \\
2 \mathrm{At}^{1} \\
2 \mathrm{At} \\
2 \mathrm{At}\end{array}$ & $\begin{array}{l}19 / 27 / 28 \\
19 / 27 / 28 \\
19 / 27 / 28 \\
\text { NT }\end{array}$ & $\begin{array}{l}\text { ABC(D)EFGI } \\
\text { ABC(D)(E)FGI } \\
\text { ABC(D)(E)FGI } \\
\text { ABCDEFGI(K) }\end{array}$ & $\begin{array}{l}\text { T11 } \\
\text { T11 } \\
\text { T11 } \\
\text { T11 }\end{array}$ & $\begin{array}{l}15 / 1 / 69 \\
15 / 1 / 69 \\
15 / 1 / 69 \\
15 / 1 / 69\end{array}$ \\
\hline B & $\begin{array}{l}\text { Nose } \\
\text { Finger } \\
\text { Sheet } \\
\text { Pillow } \\
\text { Sputum }\end{array}$ & $\begin{array}{l}30 \\
30 \\
30 \\
30 \\
30\end{array}$ & $\begin{array}{l}2 \\
2 \\
2 \\
2 \\
2\end{array}$ & $\begin{array}{l}1 \\
1 \\
1 \\
1 \\
1\end{array}$ & $\begin{array}{l}26 \\
26 w^{2} \\
26 w \\
26 w \\
26 w\end{array}$ & $\begin{array}{l}\text { ABC(D)EFH } \\
\text { AB(C)(D)(E)F(H) } \\
\text { ABC(D)EF(H) } \\
\text { ABC(D)EF(H) } \\
\text { ABC(D)(E)F(H) }\end{array}$ & $\begin{array}{l}\text { T9 } \\
\text { T9 } \\
\text { T9 } \\
\text { T9 } \\
\text { T9 }\end{array}$ & $\begin{array}{l}17 / 12 / 68 \\
18 / 12 / 68 \\
18 / 12 / 68 \\
18 / 12 / 68 \\
19 / 12 / 68\end{array}$ \\
\hline C & $\begin{array}{l}\text { Nose } \\
\text { Finger } \\
\text { Bed }\end{array}$ & $\begin{array}{l}\mathbf{3} \\
\mathbf{3} \\
\mathbf{3}\end{array}$ & $\begin{array}{l}1 \\
1 \\
1\end{array}$ & $\begin{array}{l}5 \\
5 \\
5\end{array}$ & $\begin{array}{l}9 \\
9 \\
9\end{array}$ & $\begin{array}{l}\text { ACEG } \\
\text { ACEG } \\
\text { ACEG }\end{array}$ & $\begin{array}{l}\mathbf{T} 1 \\
\mathbf{T} 1 \\
\mathbf{T} 1\end{array}$ & $\begin{array}{l}15 / 5 / 69 \\
16 / 5 / 69 \\
20 / 5 / 69\end{array}$ \\
\hline
\end{tabular}

Table V Comparison of strains isolated from different sites on the same patient

${ }^{1} \mathrm{At}=$ atypical, $\mathrm{w}=$ weak inhibition by phage, ${ }^{3} \mathrm{NT}=$ not typable 


\section{Discussion}

Since genera are likely to show major differences in their response to inhibitory chemicals, it was not possible to use the same reagents for typing $\boldsymbol{P}$. mirabilis as for E. coli (Elek and Higney, 1970). Screening of a large number of chemicals was necessary, and of 50 inhibitory agents found, most were discarded because of a failure to discriminate between strains. Ten chemicals were finally selected and provided a satisfactory typing system.

For the typing of $\boldsymbol{P}$. mirabilis by this method it was necessary to develop a medium which prevented swarming, did not inhibit growth, and contained a suitable indicator of growth. A variety of media and indicators was investigated (Kashbur, 1973) and tryptose agar with taurocholate and TTC was chosen. Interpretation was found to be easier with the spot inoculation technique, although stab inoculation allows 50 strains instead of 25 to be examined on one plate.

Other studies not described in this paper showed that increasing the concentration of the agar reduced growth as well as swarming and also caused difficulty in mixing of chemicals. Sodium taurocholate prevented swarming without altering the inhibitory pattern of any of the chemicals. As an indicator of growth, several mechanisms were investigated, namely, glucose fermentation, urease production, phenylalanine deamination, reduction of potassium tellurite, sodium hydrogen selenite, and triphenyl tetrazolium chloride. Diffuse colour changes in the medium or absence of a clear colour change eliminated most of these reactions; TTC reduction showed the most clear-cut results. Most of the chemical solutions were reasonably stable at $4^{\circ} \mathrm{C}$ for several weeks, and little advantage was found in storing at $-18^{\circ} \mathrm{C}$. The inhibitory activity of some of the chemicals, especially acriflavine and potassium tellurite, changed irrespective of the method of storage. A biological titration was found to be necessary with every series of tests to ensure reproducibility.

Resistotyping is only valid as long as resistance to one of the inhibitory chemicals does not commonly emerge in a previously sensitive strain or is not changed by the acquisition of a plasmid, eg, ' $R$ ' factor. Although it was preferable to avoid the use of disinfectants in common use in hospitals, the risk of resistance emerging to antibacterial agents other than antibiotics did not appear great. A method of typing using antiseptics alone has already been described (Delmotte, Beumer, Cotton, DekeyserDelmotte, Millett, Vanden Abeele, Von Grunigen, and Yourassowsky, 1971a and b). Resistance to quat- ernary ammonium compounds and Savlon (a $\frac{0}{\overrightarrow{0}}$ mixture of cetrimide and chlorhexidine) has been $\frac{}{-}$ reported, but is usually rapidly lost on subculture $\overrightarrow{\vec{F}}$ (Chaplin, 1952; Bassett, 1971). The other antiseptics in the test are not used to any great extent for $\frac{\mathrm{C}}{\circ}$ clinical purposes in British hospitals at the present $\frac{\bar{\sigma}}{\bar{c}}$ time.

Strains of Staph. aureus containing a plasmid which controls high penicillinase production usually show के some resistance to salts of mercury and sometimes $\overrightarrow{0}$ other metals (Moore, 1960; Novick and Roth, 1968). The introduction of an ' $R$ ' factor controlling resis- $\vec{\omega}$ tance to ampicillin, cephaloridine, carbenicillin, and kanamycin was not associated with any change in resistotype. No relationship was found between the resistotype and the antibiotic sensitivity pattern of $v$ the strains examined, but further studies on other $\mathbf{R}$ factors and other plasmids may be necessary.

Other methods of typing, serological (Lanyi, 온 1956; De Louvois, 1969), biochemical (Kippax, $\vec{c}$ 1957; Huang, 1966), bacteriocine typing (CraddockWatson, 1965), bacteriophage (France and Markham, 1966), and the Dienes method(Story, 1954;Skirrow, 1969) have been described and comparisons between $\square$ methods have been made (Burke et al, 1970; Tracy 응 and Thomson, 1972). Our comparison of a large number of strains has shown that the resistotyping method shows better differentiation than bacteriocine, bacteriophage, or serological typing. The large $\stackrel{\mathbb{Q}}{\unrhd}$ number of resistotypes found in one hospital $\overrightarrow{\vec{P}}$ suggests that most infections are endogenous in $\frac{\circ}{3}$ origin. Although cross infection is not common, occasional outbreaks require a typing method. Tests on a small number of strains of $P$. vulgaris suggest that resistotyping may also be of use as a typing method for these organisms. Resistotyping may be easily used in a routine laboratory; the only disadvantage is the preparation of chemical solutions, particularly if typing is infrequently required.

The authors are indebted to Dr M. T. Parker and $>$ Mr T. L. Pitt (Central Public Health Laboratory, Colindale) and Mr J. de Louvois (Edgware General N Hospital) for supplying strains of known phage, serological, and proticine types, and Dr E. J. L. N Lowbury (Birmingham Accident Hospital) and $\operatorname{Dr} \underset{\omega}{N}$ J. D. Williams (Dudley Road Hospital, Birmingham) for supplying further strains of $\boldsymbol{P}$. mirabilis.

\footnotetext{
References

Adler, J. L., Burke, J. P., Martin, D. F., and Finland, M. (1971). Proteus infections in a general hospital. I. Biochemical characteristics and antibiotic susceptibility of the organisms. Ann. intern. Med., 75, 517-530.

Bassett, D. C. J. (1971). The effect of pH on the multiplication of a pseudomonad in chlorhexidine and cetrimide. J. clin. Path., 24, 708-711.
} 
Burke, J. P., Adler, J. L., and Finland, M. (1970). Comparison of methods for typing Proteus mirabilis as applied in an epidemiological study of infections in a nursery for newborns. Antimicrob. Agents and Chemother., 10, 328-331.

Chaplin, C. E. (1952). Bacterial resistance to quaternary ammonium disinfectants. J. Bact., I. Abt. Orig., 63, 453-458.

Cradock-Watson, J. E. (1965). The production of bacteriocines by Proteus species. Ztbl. Bact., 196, 385-388.

Datta, N. (1968). Acquisition of antibiotic resistance by bacteria. In Recent Advances in Clinical Pathology, edited by S. C. Dyke, pp. 43-58. Churchill, London.

Delmotte, A., Beumer, J., Cotton, E., Dekeyser-Delmotte, N., Millett, M., Vanden Abbeele, K. G., Von Grünigen, W., and Yourassowsky, E. (1971a). Etude sur la sensibilité du bacille pyocyanique (Pseudomonas aeruginosa) aux antiseptiques et aux antibiotiques. I. Therapie, 26, 629-644.

Delmotte, A., Beumer, J., Cotton, E., Dekeyser-Delmotte, N. Millett, M., Vanden Abbeele, K. G., Von Grünigen, W., and Yourassowsky, E. (1971b). Étude sur la sensibilité du bacille pyocyanique aux antiseptiques et aux antibiotiques. II. Therapie, 26, 645-654.

De Louvois, J. (1969). Serotyping and the Dienes reaction on Proteus mirabilis from hospital infections. J. clin. Path., 22, 263-268.

Elek, S. D., and Higney, L. (1970). Resistogram typing-A new epidemological tool; application to Escherichia coli. J. med. Microbiol., 3, 103-110.

Elek, S. D., Davies, J. R., and Miles. R. (1973). Resistotyping of Shigella sonnei. J. med. Microbiol., 6, 329-345.

Finland, M. (1960). Treatment of pneumonia and other serious infections. New Engl. J. Med., 263, 207-221.
France, D. R., and Markham, N. P. (1968). Epidemiological aspects of Proteus infections with particular reference to phage typing. J. clin. Path., 21, 97-102.

Huang, C. T. (1966). Multitest media for rapid identification of Proteus species with notes on biochemical reactions of strains isolated from urine and pus. J. clin. Path., 19, 438-442.

Kashbur, I. M. (1973). A method of typing Proteus mirabilis based on the selective toxicity of chemical compounds. MSc Thesis, Birmingham University.

Kippax, P. W. (1957). A study of proteus infections in a male urological ward. J. clin. Path., 10, 211-214.

Lányi, B. (1956). Serological typing of Proteus strains from infantile enteritis and other sources. Acta Microbiol. Acad. scient. Hung. (Budapest) 417-428. Abstracted in Excerpta Medica, section 4. Med. Microbiol. (1957). 10, Abstract no. 1340.

Lewis, J., and Feckety, F. R., Jr. (1969). Proteus bacteraemia. Johns Hopkins med. J., 124, 151-156.

Moore, B. (1960). A new screen test and selective medium for the rapid detection of epidemic strains of Staph aureus. Lancet, 2, 453-458.

Novick, R. P., and Roth, C. (1968). Plasmid linked resistance to inorganic salts in Staphylococcus aureus. J. Bact., 95, 1335-1342.

Skirrow, M. B. (1969). The Dienes (mutual inhibition) test in the investigation of Proteus infections. J. med. Microbiol., 2, 471-477.

Story, P. (1954). Proteus infections in hospital. J. Path. Bact., 68, 55-62-

Szybalski, W. (1952). Gradient plates for the study of microbial resistance to antibiotics. Bact. Proc., p. 36.

Tracy, O., and Thomson, J. E. (1972). An evaluation of three methods of typing organisms of the genus Proteus. J. clin. Path., 25, 69-72. 